\title{
Non-isotopic Heegaard splittings of Seifert fibered spaces
}

\author{
DAVID BACHMAN \\ RYAN DERBY-TALBOT \\ APPENDIX BY R WEIDMANN
}

\begin{abstract}
We find a geometric invariant of isotopy classes of strongly irreducible Heegaard splittings of toroidal 3-manifolds. Combining this invariant with a theorem of $\mathrm{R}$ Weidmann, proved here in the appendix, we show that a closed, totally orientable Seifert fibered space $M$ has infinitely many isotopy classes of Heegaard splittings of the same genus if and only if $M$ has an irreducible, horizontal Heegaard splitting, has a base orbifold of positive genus, and is not a circle bundle. This characterizes precisely which Seifert fibered spaces satisfy the converse of Waldhausen's conjecture.
\end{abstract}

57M27; 57N10, 57M60

\section{Introduction}

The recent proof of Waldhausen's conjecture ( $\mathrm{Li}$ [7]) (see also work of Jaco and Rubinstein [6; 5]) establishes that a 3-manifold $M$ admits infinitely many non-isotopic Heegaard splittings of some genus only if $M$ contains an incompressible torus. We are interested in the converse of this statement. The only known examples of 3-manifolds that admit infinitely many non-isotopic Heegaard splittings of the same genus are given by Morimoto and Sakuma $[11 ; 10]$. However, these examples are somewhat special. In this paper, we give a complete characterization of closed, totally orientable Seifert fibered spaces that satisfy the converse of Waldhausen's conjecture.

In light of Li's result one would expect to use an essential torus when trying to distinguish isotopy classes of Heegaard splittings. To this end we have the following result, which is a weak version of Theorem 4.5.

Theorem 4.5' Let $T$ be an essential torus in an irreducible 3-manifold $M$. Suppose $H$ is a strongly irreducible Heegaard surface in $M$ whose minimal essential intersection number with $T$ is greater than two, and $H^{\prime}$ is any other Heegaard surface in $M$. If $H$ and $H^{\prime}$ meet $T$ in different slopes then they are not isotopic.

The term essential intersection number refers to the value of $|H \cap T|$ when the two surfaces are isotoped to meet in a collection of loops that are essential on both. It is 
well known that any strongly irreducible Heegaard surface can be isotoped to meet any essential surface in such a fashion.

Our primary goal is to distinguish non-isotopic splittings of Seifert fibered spaces. In this context we prove the following strengthening of Theorem $4.5^{\prime}$ :

Theorem 5.1 Let $M$ be a closed, totally orientable Seifert fibered space which is not a circle bundle with Euler number \pm 1 . Let $H$ be a strongly irreducible Heegaard surface in $M$ and $T$ be a non-separating, vertical, essential torus. Then the isotopy class of $H$ determines at most two slopes on $T$.

In particular, if three strongly irreducible Heegaard surfaces in such a Seifert fibered space meet some essential torus in different slopes then at most two of them are isotopic. This result is stronger than Theorem $4.5^{\prime}$ because there is no assumption on how many times any of these Heegaard surfaces meets the torus $T$.

Theorem 5.1 leaves open the possibility that a circle bundle over a surface may admit an irreducible Heegaard splitting that can be isotoped to meet some vertical essential torus in infinitely many slopes. The appendix, by R Weidmann, includes a proof that this phenomenon does happen. Moreover, the Heegaard splitting in this case is unique:

1.1 Theorem (Weidmann) Suppose $M$ is an orientable circle bundle over an orientable surface of positive genus. Then $M$ admits a unique irreducible Heegaard splitting up to isotopy.

In addition to this Weidmann proves in the appendix an algebraic analog of the above theorem when the Euler number is \pm 1 : Nielsen equivalence classes of the generating sets of the fundamental group of such a manifold are equivalent. Interestingly, the algebraic formulation of this theorem motivates his topological argument used to establish Theorem 1.1.

The following characterization of Seifert fibered spaces that contain an infinite collection of non-isotopic Heegaard splittings of some genus now follows from Theorems 5.1 and 1.1 .

1.2 Theorem Let $M$ be a closed, totally orientable Seifert fibered space. Then $M$ admits infinitely many non-isotopic Heegaard splittings of some genus if and only if

(1) $M$ has at least one irreducible, horizontal Heegaard splitting,

(2) $M$ has a base orbifold with positive genus, and

(3) $M$ is not a circle bundle. 
See Section 2 below for the relevant definitions.

Proof Moriah and Schultens have shown that irreducible Heegaard splittings of totally orientable Seifert fibered spaces are either vertical or horizontal [9]. It follows from this classification and results of Lustig and Moriah [8] and Schultens [12] that a Seifert fibered space can admit infinitely many non-isotopic Heegaard splittings of some genus only if it admits an irreducible, horizontal Heegaard splitting. Precisely which Seifert fibered spaces have irreducible horizontal Heegaard splittings have been classified by Sedgwick in terms of the Seifert data [13]. In particular, however, note that Moriah and Schultens had previously shown that a circle bundle can only admit an irreducible horizontal Heegaard splitting if its Euler number is \pm 1 (see [9, Corollary 0.5]).

An understanding of horizontal Heegaard splittings reveals that any infinite collection must be obtained by Dehn twists in vertical tori (see, for example, Hatcher's proof that incompressible surfaces in Seifert fibered spaces are either vertical or horizontal [3]). So the question of whether a given closed, totally orientable Seifert fibered space admits an infinite collection of non-isotopic splittings of some genus is reduced to determining when Dehn twisting a horizontal splitting in a vertical torus produces a non-isotopic splitting. This is recognized by Sedgwick in the following:

The author suspects ... that some Seifert fibered spaces will posses an infinite number of non-isotopic but homeomorphic splittings obtained by twisting a given horizontal splitting in vertical tori [13, page 178, line -2].

Suppose, then, that $\mathcal{V} \cup_{H} \mathcal{W}$ is an irreducible, horizontal Heegaard splitting of a Seifert fibered space $M$ and $T$ is a vertical torus. Assume first that $T$ separates $M$ into $X$ and $Y$. Then $T$ separates $H$ into a horizontal surface $H_{X}$ in $X$ (say) and a surface which is not horizontal in $Y$. But then $H_{X}$ is a union of fibers in a fibration of $X$ over $S^{1}$ (see Jaco [4, Theorem VI.34]). Hence, the effect of Dehn twisting $H$ about $T$ can be undone by pushing $H_{X}$ around the fibration. The conclusion is that a Dehn twist about a separating, vertical torus produces an isotopic Heegaard splitting. In particular, if the base orbifold of $M$ is a sphere then every vertical torus separates, and hence $M$ has finitely many non-isotopic Heegaard splittings in each genus.

Now assume the base orbifold of $M$ has positive genus. If $M$ is a circle bundle, then by Theorem 1.1 $M$ admits finitely many Heegaard splittings, up to isotopy. Henceforth, assume $M$ is not a circle bundle.

It follows from Theorems 2.6 and 5.1 of Moriah-Schultens [9] that in Seifert fibered spaces with positive genus base orbifold, all irreducible, horizontal Heegaard splittings are strongly irreducible. Hence, the surface $H$ is strongly irreducible. As the base 
orbifold has positive genus, we may find a pair of non-separating vertical tori $T_{1}$ and $T_{2}$ which meet in a single fiber $f$. A horizontal Heegaard surface such as $H$ meets each of these tori in loops that are transverse to $f$. Dehn twisting $H$ about $T_{2}$ has the same effect, on $T_{1}$, as Dehn twisting $H \cap T_{1}$ about $f$. Hence the new splitting surface meets $T_{1}$ in a different slope than the original splitting surface. Iterating the Dehn twist about $T_{2}$ thus produces an infinite collection of Heegaard splittings, all of which meet $T_{1}$ in distinct slopes. It now follows from Theorem 5.1 that this collection contains infinitely many non-isotopic splittings.

The authors would like to thank Cameron Gordon and Yo'av Rieck for helpful comments, and especially Richard Weidmann for providing the appendix.

\section{Definitions}

\subsection{Essential curves, surfaces and intersections}

A sphere in a 3-manifold is essential if it does not bound a ball. If a 3-manifold does not contain any essential spheres then it is said to be irreducible.

A loop $\gamma$ on a surface $F$ if called inessential if it bounds a disk in $F$ and essential otherwise. The intersection between surfaces $H$ and $T$ in a 3-manifold is compression free if the surfaces are transverse and every loop contained in their intersection is either essential or inessential on both surfaces. Their intersection is essential if every loop contained in their intersection is essential on both.

If $T$ is a torus then a slope on $T$ is the isotopy class of an essential loop. If $H$ is some other surface then the slope of $H \cap T$ is the slope of any component of $H \cap T$ which is essential on $T$. Note that this is only defined when there is such a component of $H \cap T$.

Suppose $F$ is embedded in a 3-manifold $M$. A compressing disk for $F$ is a disk $D$ such that $D \cap F=\partial D$ is essential on $F$. A surface is compressible if there is a compressing disk for it, and incompressible otherwise. A surface of positive genus in a 3-manifold is said to be essential if it is incompressible and non-boundary parallel.

\subsection{Heegaard splittings}

A handlebody is a 3-manifold which is homeomorphic to the neighborhood of a connected graph in $\mathbb{R}^{3}$. An expression of a 3-manifold $M$ as $\mathcal{V} \cup_{H} \mathcal{W}$ is called a Heegaard splitting if $\mathcal{V}$ and $\mathcal{W}$ are handlebodies. The surface $H$ is called the Heegaard surface. 
A Heegaard splitting $\mathcal{V} \cup_{H} \mathcal{W}$ is said to be reducible if there are compressing disks $V \subset \mathcal{V}$ and $W \subset \mathcal{W}$ for the surface $H$ such that $\partial V=\partial W$, and irreducible otherwise. A Heegaard splitting is said to be weakly reducible if there are similar disks $V$ and $W$ such that $V \cap W=\varnothing$, and strongly irreducible otherwise.

\subsection{Seifert fibered spaces}

A 3-manifold $M$ is a Seifert fibered space if there is a projection map $p: M \rightarrow \mathcal{O}$, where $\mathcal{O}$ is a surface and $p^{-1}(x)$ is a circle for each $x \in \mathcal{O}$. The surface $\mathcal{O}$ is called the base surface of the fibration, and inherits from $p$ a natural structure as an orbifold. If $x$ is a cone point of $\mathcal{O}$ then we say $p^{-1}(x)$ is an exceptional fiber. For all other $x$ we say $p^{-1}(x)$ is a regular fiber. A Seifert fibered space $M$ is totally orientable if it is orientable and its base orbifold $\mathcal{O}$ is orientable.

A surface in a Seifert fibered space is horizontal if it is transverse to each fiber. The following facts are known about horizontal surfaces. See, for example, Jaco [4].

(1) If a Seifert fibered space contains an essential surface with non-zero Euler characteristic then it can be made horizontal.

(2) Every Seifert fibered space with boundary contains a horizontal surface.

(3) If a totally orientable Seifert fibered space $M$ contains a connected, horizontal surface $F$ then $M$ can be obtained from $F \times I$ by identifying $F \times\{0\}$ with $F \times\{1\}$ via some homeomorphism.

(4) If a Seifert fibered space contains a horizontal surface which meets a regular fiber once, then it contains no exceptional fibers.

A Heegaard splitting $\mathcal{V} \cup_{H} \mathcal{W}$ of a Seifert fibered space $M$ is said to be horizontal if the surface $H$ can be obtained by the following construction. Let $M(f)$ denote the Seifert fibered space obtained from $M$ by removing a neighborhood of some fiber $f$. Then $M(f)$ has boundary, and can therefore be obtained from some surface $F$ with connected boundary by forming $F \times I$ and identifying $F \times\{0\}$ with $F \times\{1\}$ via some homeomorphism. Now take two parallel copies of $F$ and join them by a subannulus of $\partial M(f)$ to form $H$. Let $D$ denote a meridional disk for the solid torus attached to $M(f)$ to form $M$. The surface $H$ obtained by the above procedure will be a Heegaard surface in $M$ when $\partial D$ meets $\partial F$ in a point.

\section{Sweepouts}

Let $H$ denote a Heegaard surface in a 3-manifold $M$. Then there is a sweepout of $M$ by surfaces parallel to $H$. To be precise, there is a pair of graphs $\Sigma_{0}$ and $\Sigma_{1}$ embedded in $M$ and a continuous map $\Phi: H \times I \rightarrow M$ such that 
- $\Phi(H \times\{0\})=\Sigma_{0}$,

- $\Phi(H \times\{1\})=\Sigma_{1}$,

- there is an $s$ such that $\Phi(H \times\{s\})=H$, and

- $\Phi$ is a homeomorphism when restricted to $H \times(0,1)$.

Henceforth, we denote $\Phi(H \times\{s\})$ as $H_{S}$.

Now suppose $M$ is irreducible, $T$ is an essential torus in $M$ and $H$ is strongly irreducible. The sweepout $\Phi$ induces a height function $h: T \rightarrow I$ as follows: if $x \in T \cap H_{s}$ then $h(x)=s$. We assume $\Phi$ is chosen so that $h$ is Morse on $h^{-1}(0,1)$.

3.1 Lemma There are values $s_{-}<s_{+}$corresponding to saddle tangencies such that $H_{s} \cap T$ is compression free if and only if $H_{s}$ is transverse to $T$ and $s_{-} \leq s \leq s_{+}$.

The fact that there exists a regular value $s$ such that $H_{S} \cap T$ is compression free is a well known result, and is established here in Claims 3.2 through 3.5 of the following proof. The real content of Lemma 3.1 is that the closure of all $s$ such that $H_{S} \cap T$ is compression free is a connected interval. This is established in Claim 3.6, which is reminiscent of Bachman-Schleimer [1, Claim 6.7].

Proof For each $s \in(0,1)$ the surface $H_{s}$ separates $M$ into handlebodies $\mathcal{V}_{s}$ and $\mathcal{W}_{s}$, where $a<b$ implies $\mathcal{V}_{a} \subset \mathcal{V}_{b}$. Let $s_{0}=0,\left\{s_{i}\right\}_{i=1}^{n-1}$ the values of $s$ where $H_{s}$ is not transverse to $T$, and $s_{n}=1$.

We now label the intervals $\left[s_{i}, s_{i+1}\right]$ as follows. If, for some value of $s \in\left(s_{i}, s_{i+1}\right)$, the intersection set $H_{S} \cap T$ contains a loop which is essential on $H_{S}$ and bounds a disk in $\mathcal{V}_{s}$ then we label the interval $\left[s_{i}, s_{i+1}\right]$ with the letter " $V$ ". Similarly, if the intersection set $H_{S} \cap T$ contains a loop which is essential on $H_{S}$ and bounds a disk in $\mathcal{W}_{s}$ then we label the interval $\left[s_{i}, s_{i+1}\right]$ with the letter " $W$ ". Note that $H_{S} \cap T$ is compression free if and only if $s$ is in an unlabeled interval.

3.2 Claim For every $s \in(0,1)$ the intersection $H_{S} \cap T$ contains a loop which is essential on $H_{S}$.

Proof Suppose not. Then a standard innermost disk argument would show that $T$ may be isotoped to be disjoint from $H_{S}$, and hence lie in a handlebody. This is a contradiction, as $T$ is incompressible.

3.3 Claim The interval $\left[s_{0}, s_{1}\right]$ is labeled " $V$ " and the interval $\left[s_{n-1}, s_{n}\right]$ is labeled " $W$ ". 
Proof Choose some $s$ just larger than $s_{0}=0$. Then $H_{S}$ meets $T$ in a collection of loops which all bound disks in $\mathcal{V}_{s}$. By the previous claim at least one of these loops is essential on $H_{s}$, so the interval $\left[s_{0}, s_{1}\right]$ is labeled " $V$ ". A symmetric argument completes the proof.

3.4 Claim No interval is labeled with both a " $V$ " and a " $W$ ".

Proof Suppose this is the case for the interval $\left[s_{i}, s_{i+1}\right]$. Choose some $s \in\left(s_{i}, s_{i+1}\right)$. Then there are loops in $H_{s} \cap T$ bounding disks in $\mathcal{V}_{s}$ and $\mathcal{W}_{s}$. This contradicts the strong irreducibility of $H_{s}$.

3.5 Claim Intervals with the labels " $V$ " and " $W$ " cannot be adjacent.

Proof Suppose $\left[s_{i-1}, s_{i}\right]$ and $\left[s_{i}, s_{i+1}\right]$ are adjacent intervals with different labels. Then the surface $H_{s_{i}}$ meets $T$ in a saddle tangency. Let $\Omega$ denote the graph $H_{s_{i}} \cap T$ and $N(\Omega)$ a regular neighborhood of this graph on $H_{S_{i}}$.

Without loss of generality assume the label of $\left[s_{i-1}, s_{i}\right]$ is " $V$ ". For small $\epsilon$ the intersection $H_{s_{i}-\epsilon} \cap T$ contains a loop bounding a disk in $\mathcal{V}_{s_{i}-\epsilon}$ (say), so there is a loop of $\partial N(\Omega)$ bounding a disk in $\mathcal{V}_{s_{i}}$. Similarly, $H_{s_{i}+\epsilon} \cap T$ contains a loop bounding a disk in $\mathcal{W}_{s_{i}+\epsilon}$, so there is a loop $\partial N(\Omega)$ bounding a disk in $\mathcal{W}_{s_{i}}$. As these loops are either the same or are disjoint we again contradict strong irreducibility.

It follows from the preceding claims that there exists an unlabeled interval. The proof of the lemma is then complete once we establish the following:

3.6 Claim The union of the unlabelled intervals is connected.

Proof Suppose $\left[s_{i}, s_{i+1}\right],\left[s_{j}, s_{j+1}\right]$ and $\left[s_{k}, s_{k+1}\right]$ are intervals where $i<j<k$, $\left[s_{i}, s_{i+1}\right]$ and $\left[s_{k}, s_{k+1}\right]$ are unlabeled, and $\left[s_{j}, s_{j+1}\right]$ has a label. Without loss of generality assume the label of $\left[s_{j}, s_{j+1}\right]$ is " $V$ ". Then there is a disk $V \subset \mathcal{V}_{s}$ such that $\partial V=\alpha \subset H_{S} \cap T$ for some $s \in\left(s_{j}, s_{j+1}\right)$. As $T$ is incompressible an innermost disk argument can be used to show that the loop $\alpha$ bounds a disk $V^{\prime} \subset T$.

Choose $s^{\prime} \in\left(s_{i}, s_{i+1}\right)$. As $i<j$ we have $s^{\prime}<s$. We claim that $V^{\prime} \cap H_{s^{\prime}}$ contains a loop which is essential on $H_{s^{\prime}}$. If not then an innermost disk argument would show that $V^{\prime}$ can be isotoped to be disjoint from $H_{s^{\prime}}$. Now let $\alpha^{\prime}$ denote a loop of $V^{\prime} \cap H_{s}$ which is innermost (on $V^{\prime}$ ) among all loops which are essential on $H_{S}$ (possibly $\alpha^{\prime}=\alpha$ ). Let $V^{\prime \prime}$ denote the subdisk of $V^{\prime}$ bounded by $\alpha^{\prime}$. Then an innermost disk argument shows that $V^{\prime \prime}$ can be isotoped to a compressing disk for $H_{S}$, while still being disjoint from $H_{s^{\prime}}$. As the region between $H_{s}$ and $H_{s^{\prime}}$ is a product it follows that $V^{\prime \prime} \subset \mathcal{W}_{s}$. 
We conclude $\alpha$ is a loop of $H_{S}$ bounding a compressing disk in $\mathcal{V}_{s}$ and $\alpha^{\prime}$ is a loop bounding a compressing disk in $\mathcal{W}_{s}$, contradicting the strong irreducibility of $H_{s}$.

We conclude that $V^{\prime} \cap H_{s^{\prime}}$ contains a loop which is essential on $H_{S^{\prime}}$. Let $\beta$ denote such a loop which is innermost (on $V^{\prime}$ ). Note that as $V^{\prime} \subset T$ we have $\beta \subset H_{s^{\prime}} \cap T$. Since the interior of the subdisk of $T$ bounded by $\beta$ meets $H_{S^{\prime}}$ in loops that are inessential on both surfaces we may remove them by an innermost disk argument. Hence, $\beta$ bounds a compressing disk for $H_{s^{\prime}}$, which must lie in either $\mathcal{V}_{s^{\prime}}$ or $\mathcal{W}_{s^{\prime}}$. In either case the interval $\left[s_{i}, s_{i+1}\right]$ would have had a label.

If, initially, the label of $\left[s_{j}, s_{j+1}\right]$ was " $W$ " we would have chosen $s^{\prime} \in\left(s_{k}, s_{k+1}\right)$ and used a symmetric argument.

\section{Compression free isotopies}

4.1 Definition Let $T$ be an essential torus in a 3-manifold $M$. An isotopy $H \times I \rightarrow$ $M$ is compression free with respect to $T$ if, for all $t \in I$ such that $H_{t}$ is transverse to $T$, the intersection $H_{t} \cap T$ is compression free.

4.2 Lemma Let $H_{0}$ and $H_{1}$ denote isotopic, strongly irreducible Heegaard surfaces in an irreducible 3-manifold $M$. Let $T$ be an essential torus in $M$. Suppose $H_{i} \cap T$ is compression free, for $i=0,1$. Then there is an isotopy from $H_{0}$ to $H_{1}$ which is compression free with respect to $T$. Furthermore, there is such an isotopy such that the tangencies of $H_{t} \cap T$ which develop are either centers, saddles, or double-saddles.

Proof Let $H_{t}$ denote any isotopy from $H_{0}$ to $H_{1}$. We now define a two-parameter family of Heegaard surfaces. Note that for each $t$ the surface $H_{t}$ defines a sweepout $\Phi_{t}: H_{t} \times I \rightarrow M$. We denote $\Phi_{t}\left(H_{t} \times\{s\}\right)$ as $H_{(t, s)}$. This defines a map from $H \times I \times I$ into $M$, which we can choose to be continuous in $s$ and $t$. Furthermore, there are values $s_{0}$ and $s_{1}$ such that $H_{\left(0, s_{0}\right)}=H_{0}$ and $H_{\left(1, s_{1}\right)}=H_{1}$.

Now suppose $T$ is an essential torus in $M$. Let $\Gamma$ denote the set of points in $I \times I$ such that $H_{(t, s)}$ is not transverse to $T$. According to Cerf theory [2] we may assume $\Gamma$ is a graph with vertices of valence two and four, and for each $t$ at most one vertex of $\Gamma$ is contained in $t \times I$. We say $t$ is a regular value if there is no vertex of $\Gamma$ in $t \times I$.

Let $S$ denote the closure of the set of points $(t, s) \subset I \times I$ such that $H_{(t, s)} \cap T$ is compression free. We now claim that there is a path from $\left(0, s_{0}\right)$ to $\left(1, s_{1}\right)$ in $S$. Such a path defines the desired compression free isotopy from $H_{0}$ to $H_{1}$. It may pass through edges of $\Gamma$ corresponding to center or saddle tangencies, or a valence four vertex of $\Gamma$ which will correspond to two saddle tangencies. 
Let $\pi: I \times I \rightarrow I$ denote projection onto the first factor. Let $p$ and $q$ denote paths in $S \subset I \times I$ (ie, embedded intervals) such that
(1) $\left(0, s_{0}\right) \in p$,
(2) $\left(1, s_{1}\right) \in q$,
(3) the lengths of $\pi(p)$ and $\pi(q)$ are maximal.

4.3 Claim If the sum of the lengths of $\pi(p)$ and $\pi(q)$ is greater than one then there is a path in $S$ from $\left(0, s_{0}\right)$ to $\left(1, s_{1}\right)$.

Proof In this case there is an $x \in p$ and a $y \in q$ such that $\pi(x)=\pi(y)$ is a regular value of $t$. By Lemma 3.1 the subinterval $r$ of $\pi(x) \times I$ connecting $x$ to $y$ is in $S$. Let $p^{\prime}$ denote the subpath of $p$ connecting $\left(0, s_{0}\right)$ to $x$ and $q^{\prime}$ the subpath of $q$ connecting $y$ to $\left(1, s_{1}\right)$. Then the path $p^{\prime} \cup r \cup q^{\prime}$ is the desired path from $\left(0, s_{0}\right)$ to $\left(1, s_{1}\right)$.

4.4 Claim The lengths of $\pi(p)$ and $\pi(q)$ are equal to one.

Proof By way of contradiction, assume the length of $\pi(p)$ is less than one. Let $\left(t^{*}, s^{*}\right)$ denote the endpoint of $p$ which is not $\left(0, s_{0}\right)$.

For each $t$ there is at most one vertex of $\Gamma$ in $t \times I$. We may thus choose an $\epsilon$ small enough so that there is at most one vertex of $\Gamma$ in the rectangle $R=\left[t^{*}-\epsilon, t^{*}+\epsilon\right] \times I$. Let $t^{-}=t^{*}-\epsilon$ and $t^{+}=t^{*}+\epsilon$. We may assume that $t^{-}$and $t^{+}$are regular values of $t$. Finally, as $\epsilon$ is chosen to be small we may assume that there is at most one component of $\Gamma \cap R$ which is not an arc connecting $t^{-} \times I$ to $t^{+} \times I$.

Let $p^{\prime}$ denote the closure of $p \backslash R$. Let $x$ denote the endpoint of $p^{\prime}$ which is not $\left(0, s_{0}\right)$. Note that $\pi(x)=t^{-}$(see Figure 1). Let $S^{\prime}$ be the closure of the component of $R \backslash \Gamma$ that contains $x$. Since $x \in S$ it follows that $S^{\prime} \subset S$. If $S^{\prime}$ meets the edge $t^{+} \times I$ of $R$ then there is a path $p^{\prime \prime}$ in $S^{\prime}$ (and hence in $S$ ) from $x$ to a point of $t^{+} \times I$. The path $p^{\prime} \cup p^{\prime \prime}$ thus contradicts the maximality of the length of $\pi(p)$.

We assume then that $S^{\prime}$ does not meet $t^{+} \times I$. Let $S^{\prime \prime}$ denote the closure of a component of $R \backslash \Gamma$ which is a subset of $S$ and meets the edge $t^{+} \times I$ (such a component exists by Lemma 3.1). By Lemma 3.1 the set $S \cap\left(t^{-} \times I\right)$ is connected. Hence, if $S^{\prime \prime}$ also meets $t^{-} \times I$ then as before we can extend the path $p$ to $t^{+} \times I$, contradicting our assumption that $\pi(p)$ is maximal.

We are now reduced to the case that $S^{\prime}$ does not meet $t^{+} \times I$ and $S^{\prime \prime}$ does not meet $t^{-} \times I$. The only way in which this can happen is if $S^{\prime}$ meets $S^{\prime \prime}$ in a valence four vertex $v$ of $\Gamma$. We conclude that there is a path $p^{\prime \prime}$ which goes from $x$, through $S^{\prime}$, across $v$, through $S^{\prime \prime}$, and connects to $t^{+} \times I$. The path $p^{\prime} \cup p^{\prime \prime}$ again contradicts the maximality of the length of $\pi(p)$. 


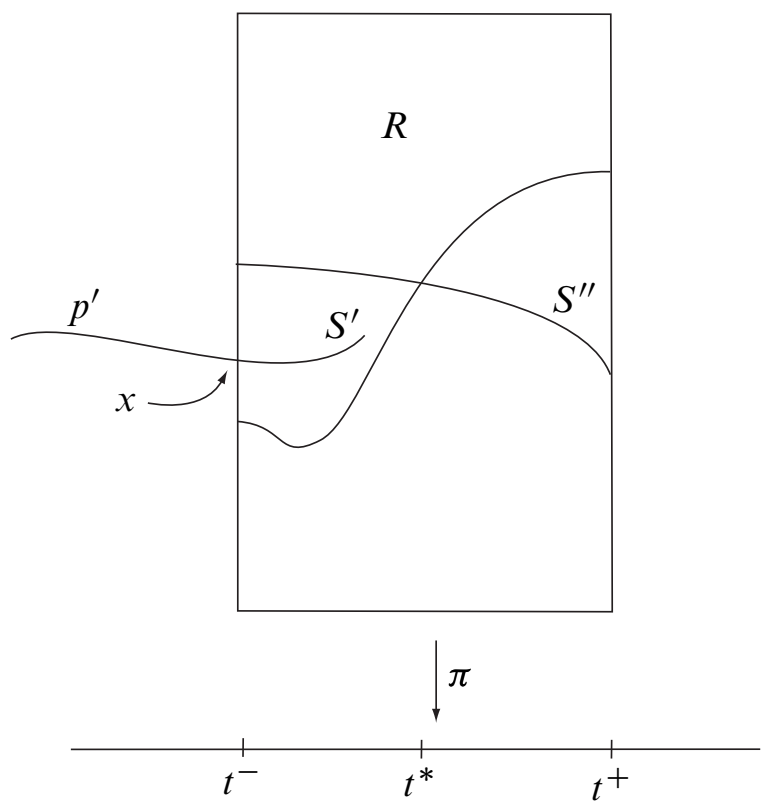

Figure 1: The rectangle $R$

The preceding claims complete the proof of Lemma 4.2.

4.5 Theorem Let $T$ be an essential torus in an irreducible 3-manifold $M$. Suppose $H_{0}$ and $H_{1}$ are isotopic, strongly irreducible Heegaard surfaces which meet $T$ essentially. Then either $H_{0}$ can be isotoped to meet a neighborhood of $T$ in a toggle or $H_{0}$ determines the same slope on $T$ as $H_{1}$.

The term toggle refers to the configuration depicted in Figure 5. It can be constructed as follows. Let $\alpha$ and $\beta$ be essential loops on $T$ which meet in a point $p$. Let $\Sigma$ be the graph $(\alpha \times\{0\}) \cup(p \times I) \cup(\beta \times\{1\})$ in $T \times I$. Then the frontier of a neighborhood of $\Sigma$ in $T \times I$ is a toggle. The word "toggle" comes from the fact that such a configuration allows one to switch back and forth between two slopes in a neighborhood of $T$.

Proof By Lemma 4.2 we know that there is a compression free isotopy from $H_{0}$ to $H_{1}$. We now discuss the various tangencies with $T$ that can develop during such an isotopy, and how they effect the slope of $H_{t} \cap T$.

Center Tangencies The simplest is a center tangency. Such tangencies only introduce or eliminate inessential loops, and hence do not change the slope of $H_{t} \cap T$. 
Saddle Tangencies The next type of tangency is a saddle. If $H^{\prime}$ is obtained from $H$ by passing through a saddle with $T$ then there is a disk $S$ such that $\partial S=\alpha \cup \beta$, where $S \cap T=\alpha$ and $S \cap H=\beta$ (see Figure 2). The surface $H^{\prime}$ is then obtained from $H$ by an isotopy guided by $S$. Hence the intersection set $H^{\prime} \cap T$ can be obtained from $H \cap T$ by a band sum along the arc $\alpha$. We call such a disk $S$ a saddle disk.

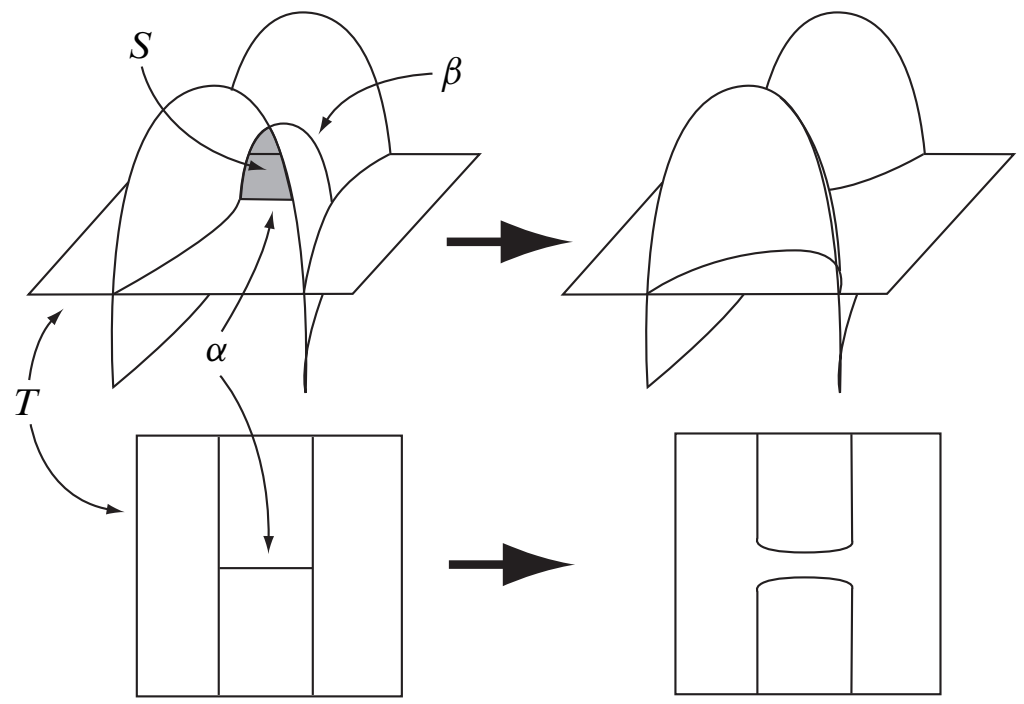

Figure 2: A saddle disk

Note that the only way that the slope of $H \cap T$ can be different from the slope of $H^{\prime} \cap T$ is if somehow all of the essential loops of $H \cap T$ were effected during the saddle move. But the only such loops that will be effected are those that contain the endpoints of $\alpha$. It follows that $H \cap T$ contains exactly two essential loops, and $\alpha$ is an arc which connects them. But then a band sum along $\alpha$ will produce an intersection set with no essential loops on $T$. This is impossible, as $H^{\prime} \cap T$ is compression free.

Double-saddle Tangencies Finally we consider what happens at double-saddles. Suppose $H^{\prime}$ is obtained from $H$ by passing through a double-saddle with $T$. Then there are two saddle disks $S_{1}$ and $S_{2}$, where $\partial S_{i}=\alpha_{i} \cup \beta_{i}, S_{i} \cap T=\alpha_{i}$, and $S_{i} \cap H=\beta_{i}$. The intersection set $H^{\prime} \cap T$ is obtained from $H \cap T$ by simultaneous band sums along $\alpha_{1}$ and $\alpha_{2}$.

In order for the slope of $H^{\prime} \cap T$ to be different from the slope of $H \cap T$ all of the essential loops of $H \cap T$ must contain an endpoint of either $\alpha_{1}$ or $\alpha_{2}$. This immediately implies $H \cap T$ contains at most four essential loops. The possibility that there are 
one or three such loops is ruled out by the fact that $H$ is separating. If there are four such loops, and each contains an endpoint of $\alpha_{1}$ or $\alpha_{2}$, then $H^{\prime} \cap T$ contains only inessential loops. This is ruled out by the fact that $H^{\prime} \cap T$ is compression free.

We conclude that if the slope of $H^{\prime} \cap T$ is different from that of $H \cap T$ then $H \cap T$ contains exactly two essential loops, $\gamma_{1}$ and $\gamma_{2}$. Up to relabeling, there are now the following cases:

(1) $\partial \alpha_{1} \subset \gamma_{1}$. Then a band sum along $\alpha_{1}$ transforms $\gamma_{1}$ into an essential loop $\gamma_{1}^{\prime}$ with the same slope on $T$, and an inessential loop $\delta$. The $\operatorname{arc} \alpha_{2}$ can either connect $\gamma_{2}$ to itself, connect $\gamma_{2}$ to $\delta$, or connect $\gamma_{2}$ to $\gamma_{1}^{\prime}$. In the first two cases a slope change does not occur. The third case implies $H^{\prime} \cap T$ contains only inessential loops, which cannot happen.

(2) Both $\alpha_{1}$ and $\alpha_{2}$ connect $\gamma_{1}$ to $\gamma_{2}$. If $\alpha_{1}$ and $\alpha_{2}$ are on the same side of $H$ then a simultaneous band sum results in all inessential loops. We conclude $\alpha_{1}$ and $\alpha_{2}$ are are on opposite sides of $H$, as in Figure 3.

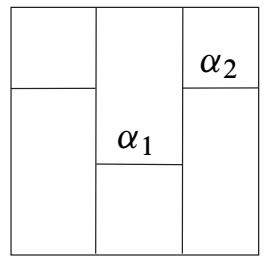

Figure 3: The set $H \cap T$ when there is a slope change at a double saddle

Now that we have narrowed down the possibilities for $H \cap T$ and $\alpha_{1}$ and $\alpha_{2}$ we must analyze the saddle disks $S_{1}$ and $S_{2}$. Before proceeding further note that if there are any inessential loops on $H \cap T$ they may be removed by an isotopy of $H$, as $H \cap T$ is compression free and $M$ is irreducible. After performing such an isotopy let $A_{i}$ be the annulus on $T$ bounded by $\gamma_{1} \cup \gamma_{2}$ containing the arc $\alpha_{i}$. Let $D_{i}$ denote the disk obtained by gluing two parallel copies of $S_{i}$ to the disk obtained from $A_{i}$ by removing a neighborhood of $\alpha_{i}$.

First note that if, for some $i$, the disk $D_{i}$ failed to be a compressing disk for $H$ it would follow that the component $H^{*}$ of $H \backslash T$ containing $\beta_{i}$ was an annulus which is parallel into $T$. Hence, a further isotopy of $H$ could push $H^{*}$ past $T$, removing all intersections of $H$ with $T$. As this is impossible, we conclude both $D_{1}$ and $D_{2}$ are compressing disks for $H$. 


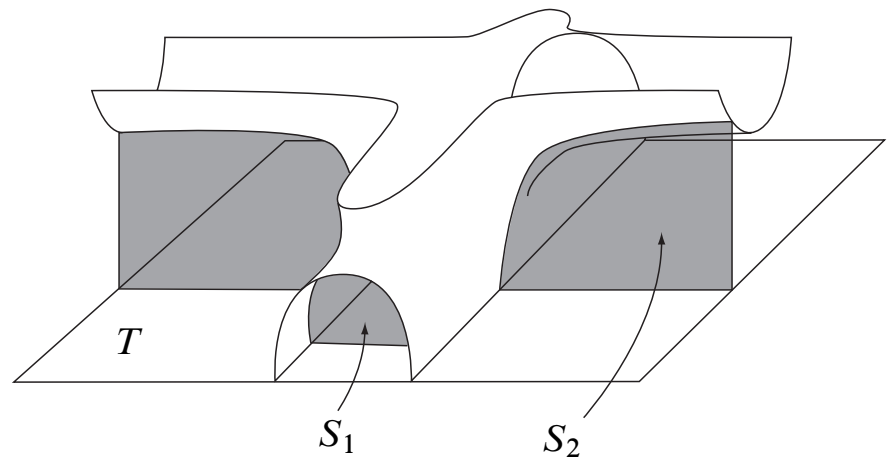

Figure 4: The surface $H \cap N(T)$

Now note that if $S_{1}$ and $S_{2}$ are incident to opposite sides of $T$ then the disks $D_{1}$ and $D_{2}$ would be disjoint. This violates the strong irreducibility of $H$. We conclude $S_{1}$ and $S_{2}$ are on the same side of $T$. Let $N(T)$ denote a copy of $T^{2} \times I$ embedded in $M$ so that $T$ is the image of $T^{2} \times\{0\}$. We may thus assume that $S_{1}$ and $S_{2}$ are contained in $N(T)$. This forces $H \cap N(T)$ to be as depicted in Figure 4. It is now an easy exercise to see that $H \cap N(T)$ is a toggle, as depicted in Figure 5.

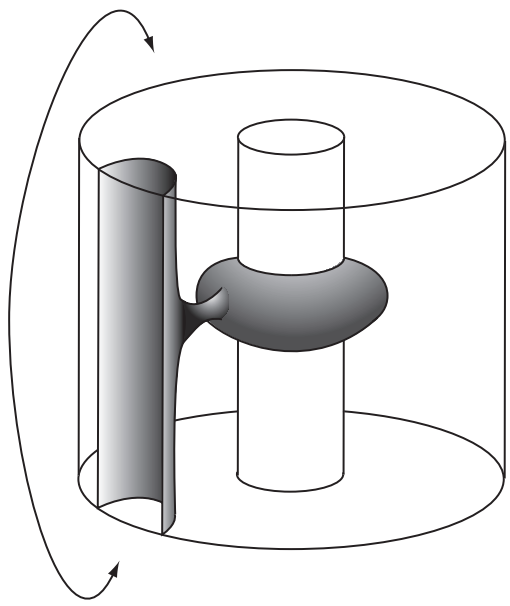

Figure 5: A toggle 


\section{Toggles in Seifert fibered spaces}

The results of the previous section leave open the possibility that if $H$ can be isotoped to meet $T$ in a toggle then $H$ may be isotoped to meet $T$ in an arbitrarily large number of slopes. In the appendix R Wedimann shows that this can, indeed, happen. Here we prove that for "most" Seifert fibered spaces it does not. In particular, we prove the following:

5.1 Theorem Let $M$ be a closed, totally orientable Seifert fibered space which is not a circle bundle with Euler number \pm 1 . Let $H$ be a strongly irreducible Heegaard surface in $M$ and $T$ be a non-separating, vertical, essential torus. Then the isotopy class of $H$ determines at most two slopes on $T$.

Remark The hypotheses of Theorem 5.1 can be relaxed to include any 3-manifold constructed in the following way. Begin with a Seifert fibered space which is not a circle bundle, with exactly two boundary components $T_{1}$ and $T_{2}$. Let $f_{i}$ denote a regular fiber on $T_{i}$. Construct $M$ by gluing $T_{1}$ to $T_{2}$ so that $\left|f_{1} \cap f_{2}\right| \neq 1$. Let $T$ denote the image of $T_{1}$ and $T_{2}$ in $M$. If $H$ is any strongly irreducible Heegaard surface in $M$ then the conclusion of Theorem 5.1 holds for the pair $(T, H)$.

Proof Let $H_{t}$ be a compression free isotopy in which there are values $t_{0}, t_{1}$ and $t_{2}$ such $H_{0}, H_{1}$ and $H_{2}$ meet $T$ in different slopes (where $H_{i}=H_{t_{i}}$ ). Assume that $t_{0}$, $t_{1}$, and $t_{2}$ are consecutive with respect to this property, in the sense that there is no value $t \in\left(t_{0}, t_{2}\right)$ such that $H_{t}$ meets $T$ in some fourth slope.

Let $N(T)$ denote a fibered, closed neighborhoood of $T$. Let $T_{\mu}$ and $T_{v}$ denote the boundary tori of $N(T)$. By Theorem 4.5 we know there is some $t_{x} \in\left(t_{0}, t_{1}\right)$ such that $H_{t_{x}}$ meets $N(T)$ in a toggle. Let $H_{x}=H_{t_{x}}$ and $\mu_{x}$ and $v_{x}$ denote the slopes of $H_{x} \cap T_{\mu}$ and $H_{x} \cap T_{\nu}$, respectively. Similarly, there is a $t_{y} \in\left(t_{1}, t_{2}\right)$ such that $H_{t_{y}}$ meets $N(T)$ in a toggle. Let $H_{y}=H_{t_{y}}$ and $\mu_{y}$ and $v_{y}$ denote the slopes of $H_{y} \cap T_{\mu}$ and $H_{y} \cap T_{\nu}$.

Let $M(T)$ denote the closure of $M \backslash N(T)$. As $H_{x} \cap N(T)$ contains compressions on both sides of $H_{x}$ (the disks $D_{1}$ and $D_{2}$ from the proof of Theorem 4.5) it follows from strong irreducibilty that $H_{x} \cap M(T)$ is incompressible in $M(T)$. By [4, Theorem VI.34] we may thus assume that each component of $H_{x} \cap M(T)$ is horizontal or vertical. Similarly, we may assume that each component of $H_{y} \cap M(T)$ is horizontal or vertical.

We now show that $H_{x} \cap M(T)$ must be horizontal. First, note that since $T$ is nonseparating $M(T)$ is connected. It follows that if $H_{x} \cap M(T)$ is not connected and one 
component is vertical then every component is vertical. This is because a horizontal component will meet every fiber, and hence will meet the fibers contained in the vertical components. We conclude the entire surface $H_{x} \cap M(T)$ is either vertical or horizontal. If it is vertical then $\mu_{x}$ and $v_{x}$ will be fibers, and hence will represent the same slope on $T$. This contradicts the fact that they are on opposite sides of a toggle.

We conclude the surface $H_{x} \cap M(T)$ is horizontal. We now assert that it consists of precisely two components, each with a single boundary component on each component of $\partial M(T)$. Suppose not. Then the surface $H_{x} \cap M(T)$ is a connected, horizontal surface. The two loops of $H_{x} \cap T_{\mu}$ inherit, from $H_{x} \cap M(T)$, orientations that agree on $T_{\mu}$. (In a totally orientable Seifert fibered space we can consistently orient each fiber. This defines a normal vector at every point of a horizontal surface.) Inspection of Figure 5 indicates that these two loops inherit, from $H_{x} \cap N(T)$, orientations that disagree. As $H_{x}$ is orientable we have thus obtained a contradiction.

A symmetric argument shows that $H_{y} \cap M(T)$ is a horizontal surface, made up of two components, each with one boundary component on each component of $\partial M(T)$. Any two horizontal surfaces in a Seifert fibered space differ by Dehn twists in vertical annuli and tori. (This is because given a spine $\Sigma$ of the base orbifold $\Sigma \times S^{1}$ cuts $M(T)$ into solid tori. As a horizontal surface intersects each such solid torus in meridian disks the only ambiguity arises from gluing the solid tori back together along vertical tori and annuli.) A Dehn twist in a vertical torus, however, does not change the boundary slopes of the surface. Similarly, as $M(T)$ is totally orientable a Dehn twist in a vertical annulus that has both boundary components on the same component of $M(T)$ will not change boundary slopes. We conclude that the pair $\left(\mu_{y}, \nu_{y}\right)$ can be obtained from the pair $\left(\mu_{x}, v_{x}\right)$ by Dehn twisting in annuli that have each of their boundary loops on different components of $\partial M(T)$. In other words, $\left(\mu_{y}, v_{y}\right)$ can be obtained from $\left(\mu_{x}, v_{x}\right)$ by simultaneous Dehn twisting along fibers. It follows that if $\mu_{x}=\mu_{y}$ then $v_{x}=v_{y}$, which is not the case, since by assumption there are exactly three distinct slopes among $\mu_{x}, \mu_{y}, v_{x}$, and $v_{y}$.

We conclude, then, that $\mu_{x}=v_{y}$ or $\mu_{y}=v_{x}$. Without loss of generality assume the former. Now note that $\mu_{y}$ meets $v_{y}$ in a point, as one is obtained from the other by passing a toggle across $T$. Finally, this implies $\mu_{x}$ meets $\mu_{y}$ in a point. But $\mu_{y}$ is obtained from $\mu_{x}$ by Dehn twisting along a fiber. This can only happen if $\mu_{x}$ (and $\mu_{y}$ ) meets each fiber once. We conclude that each component of the horizontal surface $H_{x} \cap M(T)$ meets a regular fiber once, and hence $M(T)$ has no exceptional fibers (see Section 2.3 above).

Finally, note that $M$ can be recovered from $M(T)$ by identifying its boundary components. But this must be done in such a way so that $\mu_{x}$ and $v_{x}$ meet in a point. Since 
these loops are at the boundary of a horizontal surface in $M(T)$, it must be the case that the Euler number of $M$ is positive or negative one.

\section{Appendix A Irreducible Heegaard splittings of circle bundles are unique (by $\mathbf{R}$ Weidmann)}

The goal of this appendix is to prove Theorem 1.1. We denote the orientable circle bundle over the orientable surface $S_{g}$ of genus $g \geq 1$ with Euler number $e$ by $M_{g, e}$. In [9] Y Moriah and J Schultens show that all irreducible Heegaard splittings of Seifert manifolds are isotopic to horizontal or vertical Heegaard splittings. Moreover in the case of manifolds of type $M_{g, e}$ they show (see [9], Corollary 0.5) that all irreducible Heegaard splittings of $M_{g, e}$ are vertical and of genus $2 g+1$ if $e \neq \pm 1$ and horizontal of genus $2 g$ if $e= \pm 1$. They further show that in the case $e \neq \pm 1$ the vertical splitting is unique up to isotopy. To prove Theorem 1.1 it therefore suffices to show that all genus $2 g$ horizontal Heegaard splittings of $M_{g, e}$ with $e= \pm 1$ are isotopic. The algebraic analogue of this statement is:

A.1 Theorem Let $M$ be an orientable circle bundle over an orientable surface of genus $g \geq 1$ with Euler number equal to \pm 1 . Then any two generating tuples for $\pi_{1}(M)$ of cardinality $2 g$ are Nielsen equivalent.

We prove this theorem first, as the proof motivates the proof of Theorem 1.1.

Let $G$ be a group and $\mathcal{T}=\left(g_{1}, \ldots, g_{n}\right)$ and $\mathcal{T}^{\prime}=\left(g_{1}^{\prime}, \ldots, g_{n}^{\prime}\right)$ be two tuples of elements. Recall that $\mathcal{T}$ and $\mathcal{T}^{\prime}$ are called elementary equivalent if one of the following holds.

(1) There exists some $\sigma \in S_{n}$ such that $g_{i}^{\prime}=g_{\sigma(i)}$ for $1 \leq i \leq n$.

(2) $g_{i}^{\prime}=g_{i}^{-1}$ and $g_{j}^{\prime}=g_{j}$ for $j \neq i$.

(3) $g_{i}^{\prime}=g_{i} g_{j}^{\varepsilon}$ for some $i \neq j$ and $\varepsilon \in\{-1,1\}$. Furtermore $g_{k}^{\prime}=g_{k}$ for $k \neq i$.

We further say that $\mathcal{T}$ and $\mathcal{T}^{\prime}$ are Nielsen equivalent if there exists a sequence of tuples $\mathcal{T}=\mathcal{T}_{0}, \ldots, \mathcal{T}_{k}=\mathcal{T}^{\prime}$ such that $\mathcal{T}_{i-1}$ and $\mathcal{T}_{i}$ are elementary equivalent for $1 \leq i \leq k$.

Proof of Theorem A.1 Let $g \geq 1$ and $e= \pm 1$. Note that

$$
\pi_{1}\left(M_{g, e}\right)=\left\langle a_{1}, \ldots, a_{2 g}, f \mid\left[a_{1}, f\right], \ldots,\left[a_{2 g}, f\right],\left[a_{1}, a_{2}\right] \cdot \ldots \cdot\left[a_{2 g-1}, a_{2 g}\right] f^{e}\right\rangle .
$$

Let further

$$
p: \pi_{1}\left(M_{g, e}\right) \rightarrow \pi_{1}\left(S_{g}\right)=\left\langle\bar{a}_{1}, \ldots, \bar{a}_{2 g} \mid\left[\bar{a}_{1}, \bar{a}_{2}\right] \cdot \ldots \cdot\left[\bar{a}_{2 g-1}, \bar{a}_{2 g}\right]\right\rangle
$$


be the projection given by $a_{i} \mapsto \bar{a}_{i}$ and $f \mapsto 1$. Recall that ker $p=\langle f\rangle$.

Note that $\left(a_{1}, \ldots, a_{2 g}\right)$ is a generating tuple of $\pi_{1}\left(M_{g, e}\right)$. To prove Theorem A.1 it suffices to show that any generating tuple $\left(y_{1}, \ldots, y_{2 g}\right)$ of $\pi_{1}\left(M_{g, e}\right)$ is Nielsen equivalent to $\left(a_{1}, \ldots, a_{2 g}\right)$.

A theorem of Zieschang [14] states that in $\pi_{1}\left(S_{g}\right)$ any is Nielsen equivalent to $\left(\bar{a}_{1}, \ldots, \bar{a}_{2 g}\right)$. It follows that for any generating tuple $\left(y_{1}, \ldots, y_{2 g}\right)$ of $\pi_{1}\left(M_{g, e}\right)$ the tuple $\left(p\left(y_{1}\right), \ldots, p\left(y_{2 g}\right)\right)$ is Nielsen equivalent to $\left(\bar{a}_{1}, \ldots, \bar{a}_{2 g}\right)$. Thus $\left(y_{1}, \ldots, y_{2 g}\right)$ and $\left(a_{1} f^{z_{1}}, \ldots, a_{2 g} f^{z_{2 g}}\right)$ are Nielsen equivalent for some $z_{i} \in \mathbb{Z}$ for $1 \leq i \leq 2 g$.

It clearly suffices to show that for any $i=1, \ldots, 2 g$ and $\eta \in\{-1,1\}$ there exists a sequence of Nielsen equivalences that replaces the tuple $\left(a_{1} f^{z_{1}}, \ldots, a_{2 g} f^{z_{2 g}}\right)$ with $\left(a_{1} f^{z_{1}}, \ldots, a_{i-1}^{z_{i-1}}, a_{i} f^{z_{i}+\eta}, a_{i+1}^{z_{i+1}}, \ldots \ldots, a_{2 g} f^{z_{2 g}}\right)$, ie, that replaces $a_{i} f^{z_{i}}$ with $a_{i} f^{z_{i}+\eta}$ and leaves all other elements unchanged. Note first that there is a cyclic conjugate $r$ of the relator $\left[a_{1}, a_{2}\right] \cdot \ldots \cdot\left[a_{2 g-1}, a_{2 g}\right] f^{e}$ if $\eta=-e$ and of its inverse if $\eta=e$ such that (after using the fact that $f$ commutes with the $a_{i}$ )

$$
r=f^{-\eta} a_{i}^{-1} w_{1} a_{i} w_{2}
$$

where $w_{1}$ and $w_{2}$ are words in $a_{1}, \ldots, a_{i-1}, a_{i+1}, \ldots, a_{2 g}$ such that any of the $a_{j}$ $(j \neq i)$ occurs in $w_{1}$ and $w_{2}$ once with exponent +1 and once with exponent -1 . In particular we have the identity $a_{i} f^{\eta}=w_{1} a_{i} w_{2}$ in $G$.

With appropriate Nielsen moves (left and right multiplication with the elements $a_{j} f^{z_{j}}$ ) we can replace $a_{i} f^{z_{i}}$ with $w_{1} a_{i} w_{2} f^{z_{i}}$. (Note that the $f^{ \pm z_{j}}$ cancel out as every $a_{j} f^{z_{j}}$ occurs once with exponent +1 and once with exponent -1 .) As $w_{1} a_{i} w_{2} f^{z_{i}}=$ $a_{i} f^{\eta} f^{z_{i}}=a_{i} f^{z_{i}+\eta}$ this proves the claim as all these Nielsen moves have left the $a_{j} f^{z_{j}}$ with $j \neq i$ untouched.

We illustrate the main step of the above proof with an example. Suppose that $g=2$ and $e=1$ and that we want to show that

$$
\left(a_{1} f^{z_{1}}, a_{2} f^{z_{2}}, a_{3} f^{z_{3}}, a_{4} f^{z_{4}}\right)
$$

is Nielsen equivalent to

$$
\left(a_{1} f^{z_{1}}, a_{2} f^{z_{2}+1}, a_{3} f^{z_{3}}, a_{4} f^{z_{4}}\right),
$$

this is the case with $i=2$ and $\eta=1$.

Note that the inverse of the long relation from the presentation of $\pi_{1}\left(M_{2,1}\right)$ is

$$
f^{-1} a_{4} a_{3} a_{4}^{-1} a_{3}^{-1} a_{2} a_{1} a_{2}^{-1} a_{1}^{-1},
$$


a cylic conjugate is

$$
a_{2}^{-1} a_{1}^{-1} f^{-1} a_{4} a_{3} a_{4}^{-1} a_{3}^{-1} a_{2} a_{1} .
$$

As $f$ commutes with all $a_{i}$ we have the relation

$$
r=f^{-1} a_{2}^{-1} w_{1} a_{2} w_{2}
$$

with $w_{1}=a_{1}^{-1} a_{4} a_{3} a_{4}^{-1} a_{3}^{-1}$ and $w_{2}=a_{1}$. Clearly $a_{1}, a_{3}$ and $a_{4}$ all occur twice in $w_{1}$ and $w_{2}$, once with exponent +1 and once with exponent -1 .

It follows that by applying six Nielsen moves (where each takes one of the elements $a_{1} f^{z_{1}}, a_{3} f^{z_{3}}, a_{4} f^{z_{4}}$ or their inverse and multiplies the second element in the tuple from the left or right we can replace $\left(a_{1} f^{z_{1}}, a_{2} f^{z_{2}}, a_{3} f^{z_{3}}, a_{4} f^{z_{4}}\right)$ by

$$
\begin{aligned}
\left(a_{1} f^{z_{1}},\left(a_{1} f^{z_{1}}\right)^{-1}\left(a_{4} f^{z_{4}}\right)\left(a_{3} f^{z_{3}}\right)\left(a_{4} f^{z_{4}}\right)^{-1}\left(a_{3} f^{z_{3}}\right)^{-1}\right. & \\
& \left.a_{2} f^{z_{2}}\left(a_{1} f^{z_{1}}\right), a_{3} f^{z_{3}}, a_{4} f^{z_{4}}\right)
\end{aligned}
$$

All the $f^{ \pm z_{j}}$ with $j \neq 2$ cancel in the second element of the tuple, it follows that this new tuple is nothing but

$$
\begin{aligned}
& \left(a_{1} f^{z_{1}}, a_{1}^{-1} a_{4} a_{3} a_{4}^{-1} a_{3}^{-1} a_{2} f^{z_{2}} a_{1}, a_{3} f^{z_{3}}, a_{4} f^{z_{4}}\right) \\
& \begin{aligned}
=\left(a_{1} f^{z_{1}}, w_{1} a_{2} w_{2} f^{z_{2}}, a_{3} f^{z_{3}}, a_{4} f^{z_{4}}\right) & =\left(a_{1} f^{z_{1}}, a_{2} f \cdot f^{z_{2}}, a_{3} f^{z_{3}}, a_{4} f^{z_{4}}\right) \\
& =\left(a_{1} f^{z_{1}}, a_{2} f^{z_{2}+1}, a_{3} f^{z_{3}}, a_{4} f^{z_{4}}\right)
\end{aligned}
\end{aligned}
$$

Proof of Theorem 1.1 As mentioned above, to prove Theorem 1.1 it suffices to show that any two genus $2 g$ horizontal Heegaard splittings of $M=M_{g, e}$ with $e= \pm 1$ are isotopic. We illustrate our proof of this assertion in Figure 6, in the case where the base orbifold of $M$ is a torus. The higher genus case is more difficult to see.

Let $f$ be a fiber of $M$. Note that $\hat{M}=M-N(f) \approx S_{g}^{*} \times S_{1}$ where $S_{g}^{*} \subset S_{g}$ is the once punctured orientable surface of genus $g$.

Let further $\alpha_{1}, \ldots \alpha_{2 g} \subset S_{g}^{*}$ be a canonical system of curves of $S_{g}$ with common base point $x$. Thus $\Gamma=\cup \alpha_{i}$ is a wedge of $2 g$ circles and $S_{g}-\Gamma$ is a disk. Clearly we can assume that $\bar{a}_{i}=\left[\alpha_{i}\right]$ for $1 \leq i \leq 2 g$ if

$$
\pi_{1}\left(S_{g}, x\right)=\left\langle\bar{a}_{1}, \ldots, \bar{a}_{2 g} \mid\left[\bar{a}_{1}, \bar{a}_{2}\right], \ldots,\left[\bar{a}_{2 g-1}, \bar{a}_{2 g}\right]\right\rangle .
$$

Let now $S$ be a horizontal Heegaard surface of genus $2 g$. After an isotopy of $S$ we can assume that $S$ is horizontal at the fibre $f$. Thus we can assume that $M=V \cup_{S} W$ where $V$ is the regular neighborhood in $\widehat{M}$ of a horizontal surface. In particular there exists a Heegaard graph $\hat{\Gamma}$ (a core of $V$ ) that gets mapped homeomorphically to $\Gamma$ under the projection map $\pi: \hat{M} \rightarrow S_{g}^{*} \subset S_{g},(x, z) \mapsto x$, in particular $\widehat{\Gamma}$ is a wedge 

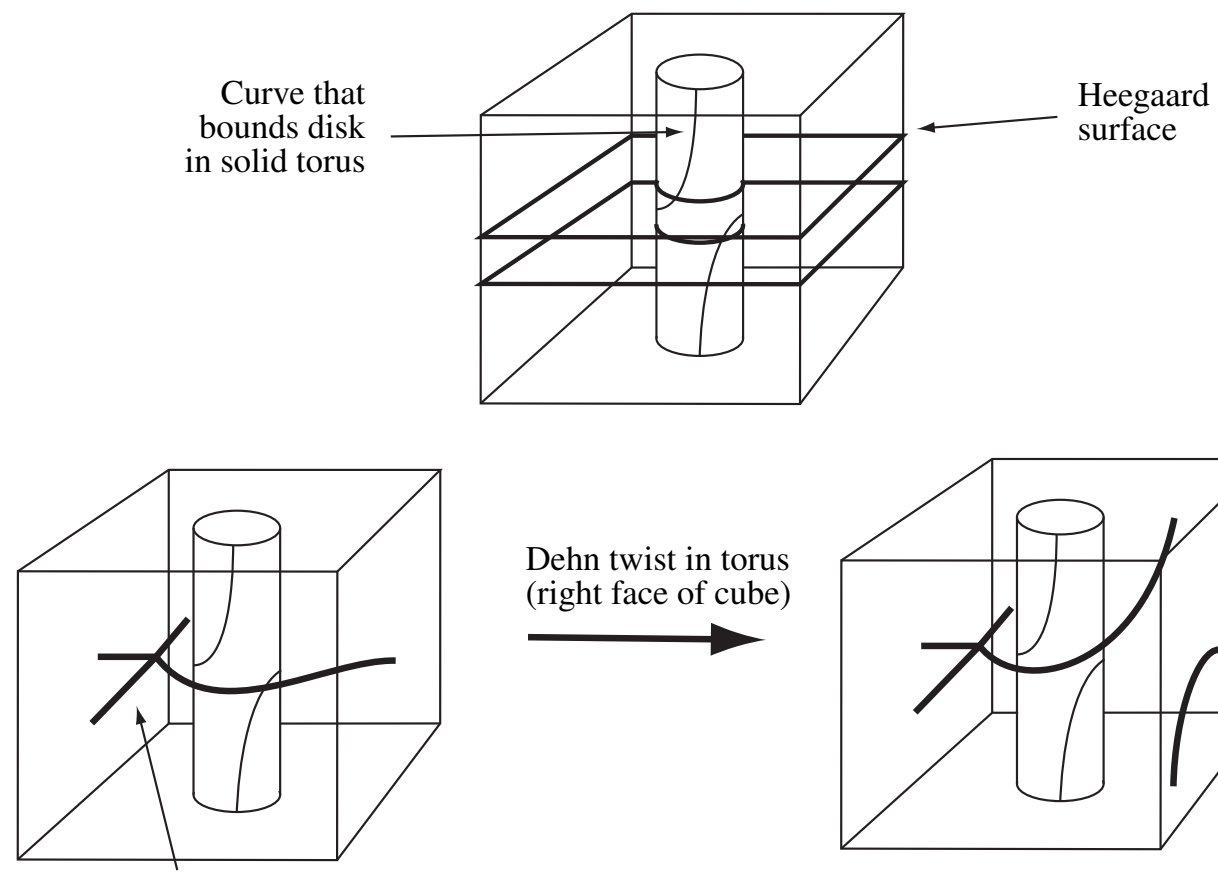

Spine of Heegaard splitting

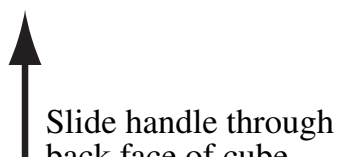

back face of cube

Dehn twist in torus (right face of cube)

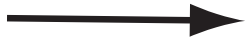

Pass spine across solid torus
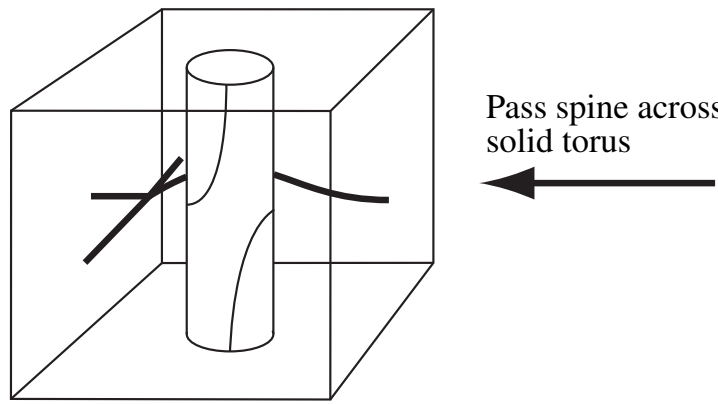

Figure 6: In $M_{1, \pm 1}$ Dehn twisting a horizontal Heegaard splitting about a vertical torus produces an isotopic splitting. 
of $2 g$ circles with single vertex $\hat{x}$ of valence greater than 2 . Denote the arc of $\widehat{\Gamma}$ that gets mapped to $\alpha_{i}$ by $\beta_{i}$.

It is clear that the (ambient) isotopy class and in fact the homotopy class of such a graph $\hat{\Gamma}$ is determined by the homotopy classes $\left[\beta_{i}\right]=a_{i} f^{z_{i}}$ of the $\beta_{i}$ in

$$
\pi_{1}(M, \hat{x})=\left\langle a_{1}, \ldots, a_{2 g}, f \mid\left[a_{1}, f\right], \ldots,\left[a_{2 g}, f\right],\left[a_{1}, a_{2}\right] \cdot \ldots \cdot\left[a_{2 g-1}, a_{2 g}\right] f^{e}\right\rangle .
$$

In particular we have a one to one correspondence between these types of Heegaard graphs and generating tuples $\left(a_{1} f^{z_{i}}, \ldots, a_{2 g} f^{z_{2 g}}\right)$ of $\pi_{1}(M, \hat{x})$.

As in the proof of Theorem A.1 it suffices to show that for any $i$ and $\eta \in\{-1,1\}$ the Heegaard graph $\widehat{\Gamma}_{1}$ corresponding to the tuple $\left(a_{1} f^{z_{i}}, \ldots, a_{2 g} f^{z_{2 g}}\right)$ of $\pi_{1}(M, \hat{x})$ is isotopic to the graph $\widehat{\Gamma}_{2}$ corresponding to the tuple

$$
\left(a_{1} f^{z_{1}}, \ldots, a_{i-1}^{z_{i-1}}, a_{i} f^{z_{i}+\eta}, a_{i+1}^{z_{i+1}}, \ldots \ldots, a_{2 g} f^{z_{2 g}}\right) .
$$

Choose a relation $r=f^{-\eta} a_{i}^{-1} w_{1} a_{i} w_{2}$ as in the proof of Theorem A.1. Recall that $\left[\beta_{j}\right]=a_{j} f^{z_{j}}$ for $1 \leq j \leq 2 g$. Let $\widehat{w}_{1}$ and $\widehat{w}_{2}$ be the words obtained from $w_{1}$ and $w_{2}$ by replacing every occurence of $a_{j}^{ \pm 1}$ by $\left[\beta_{j}\right]^{ \pm 1}$. As the fibre commutes with all $a_{1}$ it follows that we have the relation

$$
\widehat{r}=f^{-\eta}\left[\beta_{i}\right]^{-1} \widehat{w}_{1}\left[\beta_{i}\right] \widehat{w}_{2} .
$$

Let $\bar{w}_{1}$ and $\bar{w}_{2}$ be the path in $M_{g, e}$ obtained from $w_{1}$ and $w_{2}$ by replacing $a_{i}$ with $\beta_{i}$. Let further $\bar{f}$ be the fibre over $x$, clearly $[\bar{f}]=f$.

We have $\left[\bar{w}_{i}\right]=\widehat{w}_{i}$ for $i=1,2$. Now there exists a map $h: D \rightarrow M_{g, e}$ of a disk $D$ such that $h(\partial D)$ is the path $\bar{f}^{-\eta} \beta_{i}^{-1} \bar{w}_{1} \beta_{i} \bar{w}_{2}$, such that $h$ is injective on the interior $D_{0}$ of $D$ and the projection onto the base space maps $h\left(D_{0}\right)$ homeomorphically onto $S_{g}-\Gamma$.

To see this note that any lift $\tilde{\gamma}$ of $\gamma=\bar{f}^{-\eta} \beta_{i}^{-1} \bar{w}_{1} \beta_{i} \bar{w}_{2}$ to the universal covering of $M$ is a simple closed curve that is contained in the boundary of the closure $\bar{N}$ of some component $\tilde{N} \approx D^{2} \times \mathbb{R}$ of the preimage of $N=M-\Gamma \times S_{1} \approx D^{2} \times S^{1}$ under the covering map. Clearly $\tilde{\gamma}$ bounds a properly embedded disk $D$ in $\bar{N}$. Note that $\tilde{\gamma}$ is transverse to the induced foliation of $\bar{N}$ by lines except in the subpath which is the lift of $\bar{f}^{-\eta}$. This subpath is contained in a leaf of the foliation. It follows that $D$ can be chosen to be horizontal in its interior, ie, transverse to the foliation. In particular the interior of $D$ intersects every line of the foliation of $\widetilde{N}$ (the intrior of $\bar{N}$ ) exactly once. If follows that the restriction of the covering projection to $D$ is the desired map $h$.

We can now slide the edge $\beta_{i}$ over $\bar{w}_{1}$ and $\bar{w}_{2}$ which yields a new edge homotopic to $\beta_{i} \bar{f}^{\eta}$. No other edge is moved and the new edge can again be isotoped to map to $\alpha_{i}$. 


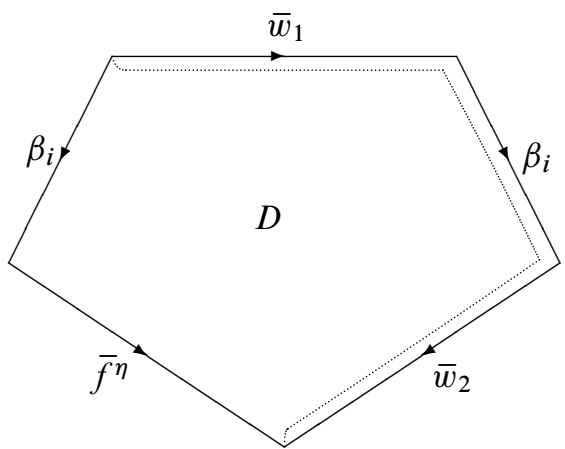

This proves the claim as the homotopy class of the new edge is $\left[\beta_{i} \bar{f}^{\eta}\right]=\left[\beta_{i}\right]\left[\bar{f}^{\eta}\right]=$ $a_{i} f^{z_{i}} f^{\eta}=a_{i} f^{z_{i}+\eta}$.

\section{References}

[1] D Bachman, S Schleimer, Surface bundles versus Heegaard splittings, Comm. Anal. Geom. to appear

[2] J Cerf, Sur les difféomorphismes de la sphère de dimension trois $\left(\Gamma_{4}=0\right)$, Lecture Notes in Mathematics 53, Springer, Berlin (1968) MR0229250

[3] A Hatcher, Notes on Basic 3-Manifold Topology Available at http:// www1. math. cornell. edu/ hatcher/3M/3Mdownloads.html

[4] W Jaco, Lectures on three-manifold topology, CBMS Regional Conference Series in Mathematics 43, American Mathematical Society, Providence, R.I. (1980) MR565450

[5] W Jaco, J H Rubinstein, 1-efficient triangulations of 3-manifolds, In preparation MR2057531

[6] W Jaco, J H Rubinstein, 0-efficient triangulations of 3-manifolds, J. Differential Geom. 65 (2003) 61-168 MR2057531

[7] $\mathbf{T} \mathbf{L i}$, Heegaard surfaces and measured laminations, I: the Waldhausen conjecture, preprint arXiv:math.GT/0408198

[8] M Lustig, Y Moriah, Nielsen equivalence in Fuchsian groups and Seifert fibered spaces, Topology 30 (1991) 191-204 MR1098913

[9] Y Moriah, J Schultens, Irreducible Heegaard splittings of Seifert fibered spaces are either vertical or horizontal, Topology 37 (1998) 1089-1112 MR1650355

[10] K Morimoto, M Sakuma, On unknotting tunnels for knots, Math. Ann. 289 (1991) 143-167 MR1087243

[11] M Sakuma, Manifolds with infinitely many non-isotopic Heegaard splittings of minimal genus, preliminary report, (unofficial) proceedings of the conference on various structures on knots and their applications (Osaka City University) (1988) 172-179 
[12] J Schultens, The stabilization problem for Heegaard splittings of Seifert fibered spaces, Topology Appl. 73 (1996) 133-139 MR1416756

[13] E Sedgwick, The irreducibility of Heegaard splittings of Seifert fibered spaces, Pacific J. Math. 190 (1999) 173-199 MR1722770

[14] H Zieschang, Über die Nielsensche Kürzungsmethode in freien Produkten mit Amalgam, Invent. Math. 10 (1970) 4-37 MR0263929

Mathematics Department, Pitzer College

1050 North Mills Avenue, Claremont CA 91711, USA

Mathematics Department, The University of Texas at Austin Austin TX 78712-0257, USA

R Weidmann: Fachbereich Informatik und Mathematik Johann Wolfgang Goethe-Universität, 60054 Frankfurt, Germany

bachman@pitzer.edu, rdtalbot@math.utexas.edu, rweidman@math . uni-frankfurt.de

Received: 2 May 2005 Revised: 6 December 2005 